\title{
A new affirmative physiological prove that Starling's law is wrong: Effect of altering fluid infusion from artery to vein on the weight of the isolated hind limb of sheep
}

\author{
Ahmed N Ghanem * \\ Mansoura University, Faculty of Medicine, Egypt Retired Consultant Urologist Surgeon \& Independent Investigator No 1 \\ President Mubarak Street, Mansoura 35511, Egypt.
}

GSC Advanced Research and Reviews, 2022, 10(02), 045-052

Publication history: Received on 07 January 2022; revised on 08 February 2022; accepted on 10 February 2022

Article DOI: https://doi.org/10.30574/gscarr.2022.10.2.0048

\begin{abstract}
Introduction and objective: Starling assumed that the capillary works as Poiseuille's tube, and its wall is impermeable to plasma proteins. Hence, fluid transfer across the capillary wall is dependent upon a balance between the capillary hydrostatic pressure and plasma protein oncotic pressure. This study aimed to verify if starling's law is correct by testing if the capillary works as the Poiseuille's tube or the new porous orifice (G) tube akin to a capillary ultrastructure anatomy.
\end{abstract}

Material and methods: A physiological study on the hind limb of sheep was conducted using both plasma proteins and crystalloid solutions. Each solution was run 3 times once through the artery then through the vein and back through the artery again while monitoring for oedema formation.

Results: Oedema only occurred when the fluid is run through the vein and not through the artery irrespective of the fluid used. This indicates that the capillary function as the G tube not Poiseuille's tube and plasma proteins passes freely through the pores hence has no oncotic effect implying that Starling's law is wrong on both forces.

Conclusion: The results affirmatively prove that the normal capillary works as G tubes not Poiseuille's tubes. Also, plasma proteins move freely between the lumen of the capillary and the ISF space-nullifying its oncotic effect. The result also proves that starling's law is wrong on both of its forces and the hydrodynamic of the capillary working as the G tube is the correct replacement for the capillary-ISF circulatory transfer.

Keywords: Capillary-ISF transfer; Starling's law; Hydrodynamics; Poiseuille's tube; Porous orifice (G) Tube

\section{Introduction and objective}

The objective of this study is to demonstrate that the capillaries work in a manner totally different from Poiseuille's tube as was proposed by Starling's hypothesis reported thrice in the Lancet in 1886 [1] and a fourth in J Physiology in 1896 [2], and hence Starling's law is wrong. Starling's hypothesis is based on a balance between two opposing forces of capillary hydrostatic pressure (CHP) and plasma oncotic pressure to induce a perfusion balance that moves fluids between the capillary lumen and the interstitial fluid (ISF) space, and back again. Starling wrongly assumed that the CHP acts in the manner demonstrated by Poiseuille in a straight uniform brass tube in which the CHP is high positive all along the tube causing filtration maximum near the inlet. Over the distal part the oncotic pressure becomes greater than CHP causing absorption again wrongly assuming that the capillary wall is impermeable to plasma proteins. Neither at the time of reporting Starling's hypothesis (1886-96) nor at the time of transferring it into a law (1948) [3] none of

\footnotetext{
${ }^{*}$ Corresponding author: Ahmed N Ghanem

Mansoura University, Faculty of Medicine, Egypt Retired Consultant Urologist Surgeon \& Independent Investigator No 1 President Mubarak Street, Mansoura 35511, Egypt.
}

Copyright (C) 2022 Author(s) retain the copyright of this article. This article is published under the terms of the Creative Commons Attribution Liscense 4.0. 
the two brilliant remarkable discoveries on the capillary ultrastructure anatomy were known yet. Both discoveries of the pre-capillary sphincter [4] and the wide inter cellular cleft pores [5] were reported in 1967. This makes the capillary a porous orifice $(G)$ tube with totally new unique hydrodynamic that is totally different from Poiseuille's tube (Figure 1 and 2).

In the light of the G tube hydrodynamic discovery summarised in diagram (Figure 3), I evaluated the effects of both forces of CHP and oncotic pressure on oedema formation on the isolated hind limb (thigh) of sheep using both changes in weight (gravimetric Method) and limb girth (volumetric method) of the isolated hind limb. The evidence that the G tube causes negative side pressure gradient along its wall that is maximum near the inlet and turns positive near the exit is demonstrated in (Figure 4). This further induce a net negative pressure in a surrounding chamber (C) (Figure 5). A plasma solution was compared to balanced isotonic crystalloid solution (Hartman or Ringer Solution) as circulating fluids in causing oedema formation. The LHP of Starling's law was also wrongly presumed to cause oedema by raising arterial and/or venous pressure alike in the study that transferred Starling's hypothesis into a law [3]. In every set of experiments monitoring of the limb oedema was obtained by measuring the thigh's girth and weight after filling the circulatory system with the fluid before the start and after finishing the experiments. A total of sex experimental sets was performed- 3 on each type of solutions.

This study aims to refute the effects of both forces of Starling's hypothesis and precise its role in oedema formation. It further proposes the $G$ tube hydrodynamic as the correct replacement for the erroneous Starling's law [6, 7]

\section{Material and methods}

The thigh of a freshly slaughtered sheep was bought from the butcher shop with adequately lengthy stump of both femoral artery and vein that was connected to an electric water pump circulating the fluid. The thigh was tightly wrapped in a cling film to replace the skin and prevent any leakage. The circulating fluid used was plasma protein and balanced crystalloid solutions in turns. In every set of experiments the connection was made to allow inflow from the artery and later swapped to allow inflow through the vein, and back to normal inflow through the artery again.

The following set of experiments were run in a consecutive order:

\section{1. $1^{\text {st }}$ set of experiments}

Plasma protein solution was used to adequately fill up the circulatory system with arterial inflow first and ran for adequate time to get rid of any air bubble and allow for stable circulation. The changes of the thigh were monitored for oedema formation by measuring the girth and weighing the limb at start and finish. The same measurements were repeated in all other experimental sets below.

\section{2. $2^{\text {nd }}$ set of experiments}

The above was repeated using plasma solution again after swabbing the inflow and outflow between artery and vein.

\section{3. $3^{\text {rd }}$ set of experiments}

The experiment was repeated on the above limb after swabbing the inflow back to arterial and the outflow to venous circulation, observing what happened to the oedema accumulated in the above 2 nd experimental set.

\section{4. $4^{\text {th }}$ set of experiments}

After evacuating the system from plasma solution and filling it up with isotonic electrolytes balanced solution (Hartmann or Ringers' solution) the $1^{\text {st }}$ set of experiments were repeated with monitoring for oedema formation.

$5^{\text {th }}$ set of experiments: After switching the inflow and outflow to vein and artery respectively, the experiment was repeated with similar measurements.

\section{5. $6^{\text {th }}$ set of experimentsF}

ollowing from the above, the circulation was switched back to normal arterial inflow and venous outflow observing what happened to the oedema accumulated in the above $5^{\text {th }}$ experimental set. 
In the $3^{\text {rd }}$ and $6^{\text {th }}$ sets of experiments where oedema manifested under the cling film samples were taken and analyzed from the sub cling film to compare plasma proteins and electrolytes concentration with a sample taken from the circulatory system.

\section{Results}

To appreciate the results of this physiological study a summary demonstrating the difference between the hydrodynamic of Poiseuille's tube (Figure 1) and that of the G tube (Figure 2) is reported here derived from physics studies on both tubes [6,7]. The lumen pressure of Poiseuille's tube is all positive causing filtration all along the entire length of the tube higher near the inlet and declines gradually but never turns negative (Figure 1). The hydrodynamic of the G tube creates a unique negative side pressure (SP) on the tube's wall maximum near the inlet that gradually declines and turns positive rising to positive maximum near the exit (Figure 2). The negative SP not only causes suction over the proximal part of the $\mathrm{G}$ tube but also induces negative pressure in a surrounding chamber (C) diagram of (Figure 3 ) that is based on pictures shown in (Figures 4 and 5). The diagram (Figure 3) demonstrating the G-C circulation simulates the capillary-ISF circulatory transfer. The physiological experiments reported here demonstrate that the real capillary does not function as Poiseuille's tube but rather works precisely as the G tube.

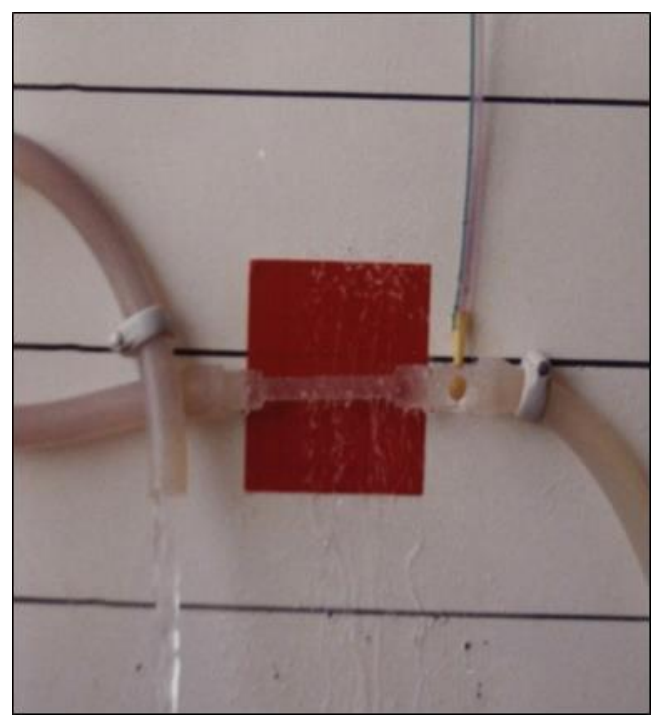

Figure 1 Poiseuille's tube hydrodynamic with positive side pressure along the entire length of the tube causing fluid to filter out maximum near the inlet and minimum near the exit. This is what Starling had based his hypothesis on regarding the hydrostatic pressure causing filtration maximum near the orifice. This will be compared to the hydrodynamic of the G tube (Figure 2) built on a scale to capillary ultrastructure of pre-capillary sphincter and intercellular clefts making wide capillary pores that allow the passage of molecules larger than plasma proteins.

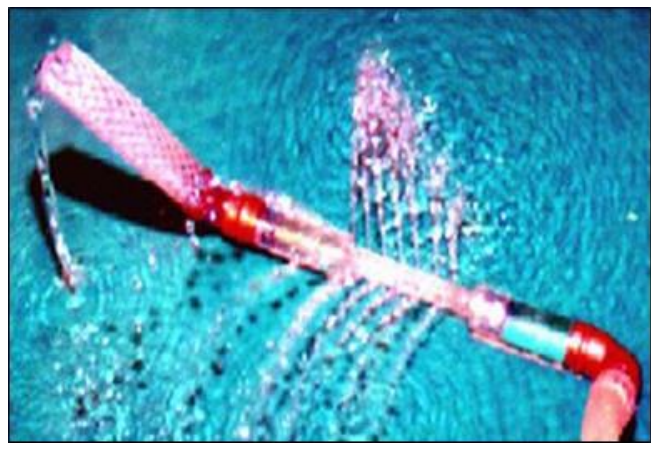


Figure 2 The hydrodynamic of the G tube with side pressure gradient lower at the inlet where it is negative and turns into positive pressure maximum near the exit, with visible magnetic field-like circulation around it seen at your top right-hand quarter of the photo-based on which and other photos shown below, the diagram showing the G-C circulation was drown (Figure 3). There is negative side pressure gradient over the proximal part of G tube not shown here but is shown in (Figure 4).

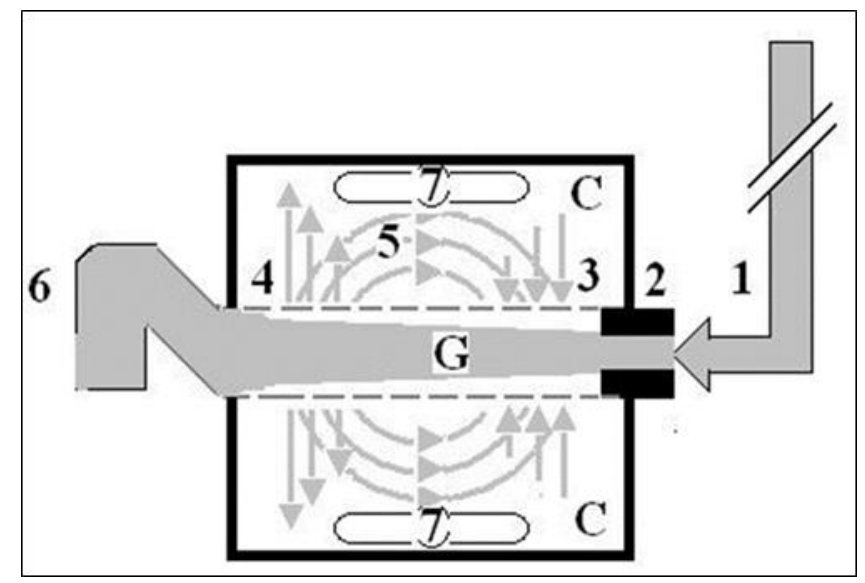

Figure 3 A diagrammatic representation of the hydrodynamic of G tube based on G tubes and chamber C seen in (Figure 5). This 37-years old diagrammatic representation of the hydrodynamic of $\mathrm{G}$ tube in chamber $\mathrm{C}$ is based on several photographs some are shown here. The $\mathrm{G}$ tube is the plastic tube with narrow inlet and pores in its wall built on a scale to capillary ultra-structure of precapillary sphincter and wide inter cellular cleft pores, and the chamber $\mathrm{C}$ around it is another bigger plastic tube representing the interstitial fluid space to form the G-C apparatus. The diagram represents a capillary-ISF unit that should replace Starling's law in every future physiology, medical and surgical textbooks, and added to chapters on hydrodynamics in physics textbooks. The numbers should read as follows

- The inflow pressure pushes fluid through the orifice.

- Creating fluid jet in the lumen of the G tube**.

- The fluid jet creates negative side pressure gradient causing suction maximal over the proximal part of the $G$ tube near the inlet that sucks fluid into lumen.

- The side pressure gradient turns positive pushing fluid out of lumen over the distal part maximally near the outlet.

- Thus, the fluid around G tube inside C moves in magnetic field-like circulation (5) taking an opposite direction to lumen flow of $\mathrm{G}$ tube.

- The inflow pressure 1 and orifice 2 induce the negative side pressure creating the dynamic G-C circulation phenomenon that is rapid, autonomous, and efficient in moving fluid and particles out from the G tube lumen at 4 , irrigating $C$ at 5 , then sucking it back again at 3 ,

- Maintaining net negative energy pressure inside chamber $\mathrm{C}$.

**Note the shape of the fluid jet inside the G tube (Cone shaped), having a diameter of the inlet on right hand side and the diameter of the exit at left hand side (G tube diameter). I lost the photo on which the fluid jet was drawn, using tea leaves of fine that can pass through the pores and coarse sizes cannot pass through the pores. Both sizes of tea leaves runs in the centre of $\mathrm{G}$ tube leaving the outer zone near the wall of $\mathrm{G}$ tube clear. This may explain the finding in real capillary of the protein-free (and erythrocyte-free) sub-endothelial zone in the Glycocalyx paradigm. It was also noted that fine tea leaves exit the distal pores in small amount maintaining a higher concentration in the circulatory system than that in the $\mathrm{C}$ chamber- akin to plasma protein 


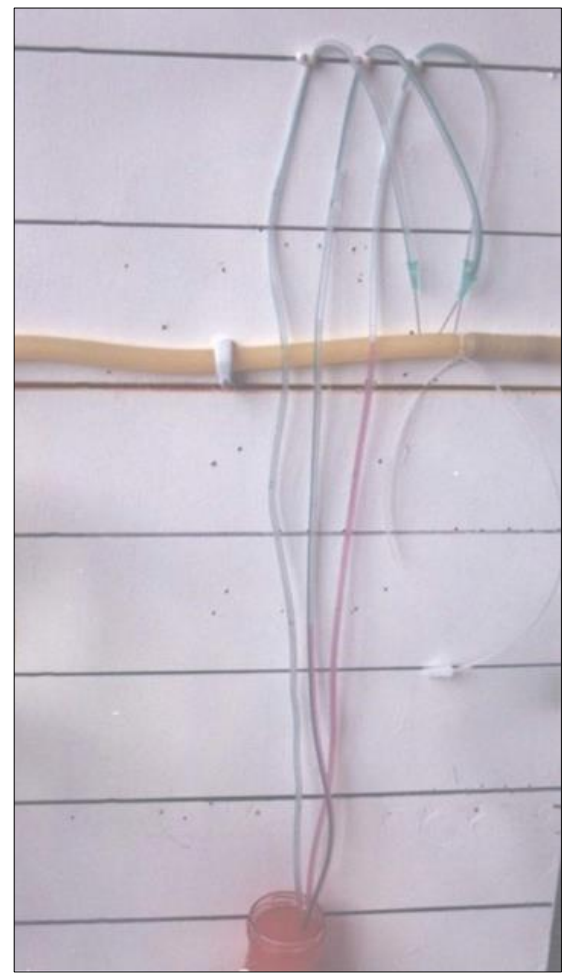

Figure 4 A rubber orifice tube's negative side pressure gradient maximum near the inlet, turning into positive pressure maximum near the exit as shown in (Figure 2), with visible magnetic field-like circulation around it seen at your top right-hand quarter of the photo- based on which and other photos, the diagram showing the G-C circulation was drown (Figure 3). This rubber orifice tube was also used for measuring the flow pressure (FP) and side pressure (SP) which are dynamic components of the lumen pressure (LP) induced by the proximal pressure (PP)- akin to arterial pressure

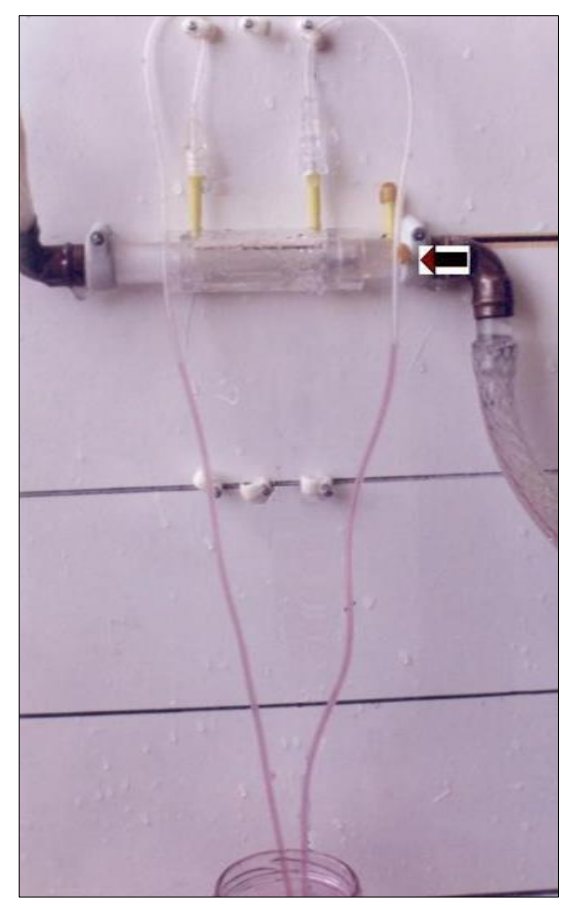

Figure 5 The G tube enclosed in chamber C (The G-C apparatus). The negative side pressure of G tube also creates a negative pressure in $\mathrm{C}$ shown here to suck the red water from a jar $300 \mathrm{~mm}$ below $\mathrm{G}$ tube into the manometers

The observations on the above 6 set of experiments were as follows: 


\section{1. $1^{\text {st }}$ set of experiments}

At the end of the experiment, the cling film was wrinkled and tightly stuck to the thigh indicating a negative subcutaneous pressure under the cling film. This indicated that the capillaries worked as G tube not Poiseuille's tube. There was no oedema formation.

\section{2. $2^{\text {nd }}$ set of experiments}

After some time running the circulation, the limb was swollen with oedema manifesting with girth and weight increase due to accumulation of fluid in the ISF space and under the cling film. The capillaries acted as Poiseuille's tube here.

\section{3. $3^{\text {rd }}$ set of experiments}

The experiment was repeated on the swollen limb above after swabbing the inflow back to arterial and the outflow to venous circulation. After some time, all the fluid in the ISF space and under the cling film was absorbed and oedema disappeared by being sucked back into the circulation restoring the limb back to normal girth and weight. This means that the capillaries' function was restored to normal acting like the G tube again and sucking all the fluid from the ISF and subcutaneous fluid space back into the circulatory system.

\section{4. $4^{\text {th }}$ set of experiments}

After evacuating the system from plasma solution and filling it up with isotonic electrolytes balanced solution (Hartmann or Ringers' solution) the $1^{\text {st }}$ set of experiments' measurements were repeated and identical results were obtained. This indicated that the capillaries worked as G tube not Poiseuille's tube. There was no oedema formation despite total absence of plasma proteins.

\section{5. $5^{\text {th }}$ set of experiments}

After switching the inflow and outflow to vein and artery respectively, the experiment was repeated with similar measurements. After some time, the limb was swollen with oedema manifesting with girth and weight increase due to accumulation of fluid (Oedema) in the ISF space and under the cling film. The capillaries acted as Poiseuille's tube here again.

\section{6. $6^{\text {th }}$ set of experiments}

Following from the above using the oedematous limb, the circulation was switched back to normal arterial inflow and venous outflow. The oedema disappeared by sucking all the excess fluid back into the circulatory system. The limb returned to normal girth and weight with the cling film tightly stuck to the flesh indicating normal capillary-ISF transfer under negative pressure meaning the capillaries working as the $\mathrm{G}$ tube again.

The plasma protein concentration in the sample taken from under the cling film of the second experimental set were identical to that taken from the circulatory system. This means that plasma protein passes freely through the capillary pores and there is no oncotic effect across the capillary membrane.

\section{Discussion}

The above reported result indicate that the capillaries work under normal conditions as the G Tube not Poiseuille's tube in which raising the arterial pressure causes suction not filtration while raising the venous pressure induces massive filtration and oedema formation. The mode of the capillary-ISF transfer is exactly as shown in the G tube diagram (Figure 3) which represent a simulation of the capillary-ISF circulatory transfer.

It is worth noting that reversing the direction of fluid flow inside the G tube makes it function like Poiseuille's tube as observed in the $2^{\text {nd }}$ and $5^{\text {th }}$ set of experiments. In Poiseuille's tube the lumen pressure is all positive causing only filtration all along the tube and inducing oedema formation irrespective of the presence or absence of plasma proteins. In the $1^{\text {st }}$ experimental set, it is wrong to assume that plasma protein is responsible for the negative pressure in the ISF space that prevent the formation of oedema. The evidence for that is the $4^{\text {th }}$ set of experiments in which only crystalloid fluid was used in total absence of plasma proteins and still no oedema occurred. The explanation of the negative ISF and subcutaneous pressure can only be attained by the $G$ tube function of the capillaries. This negative pressure in the subcutaneous space was elegantly measured by Guyton and Colman to be a minus $-7 \mathrm{~cm}$ water [8]. The capillaries may lose its normal function as $\mathrm{G}$ tube when the precapillary sphincters lose its tone, and the normal smooth inner surface of the capillaries of glycocalyx becomes irregular [9]. Both situations occur in clinical practice in sepsis and septic shock. 
The presented evidence demonstrates that both forces of Starling's law are wrong: The normal capillary tubes do not function as Poiseuille's tube, and the oncotic pressure does not work on the normal capillary membrane with wide intercellular cleft pores. I had previously reported 21 reasons why Starling's law is wrong [10]. I also documented that a gross experimental error occurred in the study that transferred Starling's hypothesis into a law $[3,7]$. The authors wrongly though that raising the capillary pressure may be achieved by raising the arterial and venous pressure alike ( $\mathrm{mmHg}$ for $\mathrm{mmHg}$ ) [3]. This is clearly not the case as demonstrated in the above results: A high venous pressure causes oedema as the capillary works as Poiseuille's tube with all positive LHP along its entire length, while high arterial pressure does not- it causes suction over the proximal part. It causes suction as the capillary works as the G tube. This should be the new scientific foundation for fluid therapy in shock management [11, 12] based on which new recommendations for the management of shock should be planned as evidence-based medicine. This should be an urgent priority to save the hundreds of thousands of patients killed per year because the faulty Starling's law dictating the faulty rules on fluid therapy for shock management [13]. It kills patients by inducing volumetric overload shocks [14] and causing ARDS [15]. The revised starling Principle is also a misnomer and wrong [16]. It is indeed time to say: "Goodbye Starling's law, hello G tube" [17].

\section{Conclusion}

A physiological study on the hind limb of sheep was conducted using both plasma proteins and crystalloid solutions and the results are presented. Each solution was run in 3 experimental sets: The inflow is run once through the artery then through the vein and back through the artery again while monitoring for oedema formation using both girth measurement and weight changes. The results affirmatively prove that the capillary works as G tube not Poiseuille's tubes. Also, plasma proteins move freely between the lumen of the capillary and the ISF space-nullifying its oncotic effect. The result of this study affirmatively proves that starling's law is wrong on both of its forces and the hydrodynamic of the capillary working as the G tube is the correct replacement for the capillary-ISF circulatory transfer.

\section{Compliance with ethical standards}

\section{Acknowledgments}

I like to thank Dr Khaled A Ghanem for his comments and criticisms.

\section{Conflict of interest}

No conflict of interest to desclosed.

\section{Funding}

None declared.

\section{References}

[1] Starling EH. Factors involved in the causation of dropsy. Lancet 1886; ii: 1266-1270, 1330-1334 and 1406-1410.

[2] Starling EH. On the absorption of fluids from connective tissue spaces. J Physiol. 1896; 19: 312-326

[3] Pappenheimer JR, Soto-Rivera A. Effective osmotic pressure of the plasma proteins and other quantities associated with the capillary circulation in the hind limbs of cats and dogs. Am J Physiol. 1948; 152: 471- 491?

[4] Rhodin J. A. The ultra-structure of mammalian arterioles and pre-capillary sphincters. J Ultrastructure Research 1967; 18: 181-222.

[5] Karnovesky M. J. The ultra-structural basis of capillary permeability studied with peroxidase as a tracer. J Cell Biol. 1967; 35: 213-236.

[6] Ghanem AN. Magnetic field-like fluid circulation of a porous orifice tube and its relevance to the capillaryinterstitial fluid circulation: preliminary report. Med Hypotheses. 2001; 56(3): 325-334.

[7] Ghanem AN. Final Affirmative Proof Starling's Law Wrong and G Tube Hydrodynamic is the Correct Replacement: New Results and Critical Analytical Criticisms of Impactful Landmark Articles. Biomed J Sci \& Tech Res. 2021; 33(5)-. BJSTR. MS.ID.005460. 
[8] Guyton AC, Coleman TG. Regulation of interstitial fluid volume and pressure. Annals New York Academy of Sciences. 1968; 150: 537-547.

[9] Ghanem AN. "Impact of COVID-19 on Surgery and Anaesthesia: Fighting another Unique War on ARDS Relevant to Covid-19 Pandemic". EC Emergency Medicine and Critical Care. 2 February 2021; 5.

[10] Ghanem AN. Twenty-one reasons affirming Starling's law on the capillary-interstitial fluid transfer wrong, and the correct replacement is the hydrodynamic of the porous orifice (G) tube. Case Rep Open An Open J. 2020; I (1): 8-11.

[11] Ghanem AN. The New Scientific Foundation of Fluid Therapy in Shock Management. Clin Surg. 2021; 6(13): 1-9.

[12] Ghanem AN. NEW SCIENTIFIC BASIS OF FLUID THERAPY IN SHOCK MANAGEMENT: THE COMPLETE EVIDENCE BASED ON NEW SCIENTIFIC DISCOVERIES IN PHYSICS, PHYSIOLOGY, AND MEDICINE. Austin Macauley Publishers Ltd $®$, London 2022. The Book comes in 35 Chapters and 516.

[13] Ghanem. Why and how Starling's law is killing patients in clinical practice in hundreds of thousands per year? Journal of Anatomy and Physiology. 2021; 2: 41-44.

[14] Ghanem AN. Volume Kinetic Shocks in Surgical Practice. J Emerg. Med Trauma Surg. Care. 2020; 2: 010.

[15] Ghanem AN. Volumetric Overload Shocks Cause the Acute Respiratory Distress Syndrome: Building the Bridge Between Physics, Physiology, Biochemistry, and Medicine. Biomed J Sci \& Tech Res. 2020; 29(1). BJSTR. MS.ID.004758.

[16] Ghanem AN. and Ghanem KA. Revised Starling's Principle (RSP): a misnomer as Starling's law is proved wrong. Med Res Chronicles. 2020.

[17] Ghanem AN. Editorial. Goodbye Starling's law, hello G tube. J Urol. Nephrol. 2020; 5(1). 